\title{
Cognitive impairment in heart failure with Cheyne-Stokes respiration
}

\author{
A D Staniforth, W J M Kinnear, A J Cowley
}

\begin{abstract}
Objectives-To document the degree of cognitive impairment in stable heart failure, and to determine its relation to the presence of Cheyne-Stokes respiration during sleep.

Subjects-104 heart failure patients and 21 healthy normal volunteers.

Methods-Overnight oximetry was used (previously validated as a screening tool for Cheyne-Stokes respiration in heart failure). Cognitive function was assessed using a battery of neuropsychological tests. Left ventricular function was assessed by echocardiography.

Results-Heart failure patients performed worse than the healthy volunteers in tests that measured vigilance. Reaction times were $48 \%$ slower $(0.89(0.03) \mathrm{s} v 0.60(0.05) \mathrm{s} ; \mathrm{p}<0.005)$ and they hit twice as many obstacles on the Steer Clear simulator (75 (6.4) v 33 (4.6); p < 0.005). Cognitive impairment within the heart failure group was unrelated to either the presence of Cheyne-Stokes respiration, the degree of left ventricular dysfunction, or indices of nocturnal oxygenation.

Conclusions-Vigilance was impaired in heart failure but this did not appear to be related to the presence of Cheyne-Stokes respiration during sleep. Impaired vigilance as measured on the Steer Clear test has been associated with an increased risk of motor vehicle accidents. The issue of fitness to drive in heart failure requires further attention.
\end{abstract}

(Heart 2001;85:18-22)

Keywords: Cheyne-Stokes respiration; cognitive function; heart failure; driving

Experiments in healthy volunteers have shown that hypoxia ${ }^{1}$ and sleep fragmentation ${ }^{2}$ lead to daytime sleepiness and cognitive impairment. Similar effects are seen in respiratory disease; indeed in obstructive sleep apnoea the effect is so profound it has been linked to a rise in motor vehicle accidents. ${ }^{3}$

Clinical experience tells us that cognitive function is impaired in heart failure. Very few experimental data exist concerning the level of cognitive impairment experienced, or its relation to sleep disordered breathing. Bornstein and colleagues documented significant levels of cognitive impairment in $50 \%$ of patients with end stage heart failure undergoing assessment for cardiac transplantation. ${ }^{4}$

The pathophysiology of cognitive impairment in heart failure is unclear. It might be related to simple pump failure and cerebral hypoperfusion. In an earlier study we showed that Cheyne-Stokes respiration was seen in $21 \%$ of patients with compensated heart failure, and that these episodes of disordered breathing were associated with blood oxygen desaturation and fragmentation of normal sleep architecture. $^{5}$ We hypothesised that the degree of daytime cognitive impairment would be related to the presence of Cheyne-Stokes respiration during sleep. The proposed mechanism behind this was either nocturnal hypoxaemia from apnoeas, or repeated arousal from sleep during the hyperpnoeic phase of Cheyne-Stokes respiration. In this paper we set out to document the degree of cognitive impairment typically experienced in medically treated stable heart failure of mild to moderate severity. We also investigated the relation between cognitive impairment and indices of nocturnal oxygen desaturation, left ventricular function, and the presence of Cheyne-Stokes respiration.

\section{Methods}

STUDY POPULATION

We recruited 104 patients from a specialist heart failure clinic over a 12 month period. Patients of any age, aetiology, and duration or severity of symptoms were included. Subjects with known pulmonary, cerebrovascular, neuromuscular, or sleep disorders were excluded. All patients had compensated heart failure with no change in drug treatment for four weeks. All received standard medical treatment (angiotensin converting enzyme (ACE) inhibitor plus diuretic). All ACE inhibitor doses were recorded as equivalent doses of captopril (captopril $150 \mathrm{mg}=$ enalapril $20 \mathrm{mg}$ $=$ lisinopril $10 \mathrm{mg}$ ). ACE inhibitor intolerant subjects were eligible for study. The study was approved by the hospital ethics committee.

Heart failure was diagnosed on the basis of medical history and examination findings, together with a visually impaired left ventricle on cross sectional echocardiography. Left ventricular function was quantified from standardised $\mathrm{M}$ mode (one dimensional) echocardiographic measurements made in the parasternal long axis. A single cardiac technician performed all examinations. Only subjects with either a left ventricular diastolic diameter greater than $5.5 \mathrm{~cm}$ or an ejection fraction less than $45 \%$ were eligible for study.

Twenty one healthy normal volunteers matched for age and body mass index (BMI) were also studied. Normal left ventricular function was confirmed by the presence of a normal 12 lead ECG and echocardiogram.

DIAGNOSIS OF CHEYNE-STOKES RESPIRATION All subjects underwent overnight oximetry in their own homes using the Ohmeda Biox 3700 pulse oximeter (Ohmeda, Colorado, USA).
Accepted 11 September 2000 
The apparatus records the lowest percentage transcutaneous oxygen saturation $\left(\mathrm{S}_{\mathrm{A}} \mathrm{O}_{2}\right)$ detected over successive 12 second time intervals for eight hours (2400 data points), and has been validated over oxygen saturation ranges of $60-98 \% .{ }^{6}$ The data were analysed to calculate the overnight minimum $\mathrm{S}_{\mathrm{A}} \mathrm{O}_{2}$, mean $\mathrm{S}_{\mathrm{A}} \mathrm{O}_{2}$, dip frequency (a dip was defined as a fall in $\mathrm{S}_{\mathrm{A}} \mathrm{O}_{2}$ $\sim 4 \%$ ), and the percentage of recording time spent with an $\mathrm{S}_{\mathrm{A}} \mathrm{O}_{2}$ of $\leqslant 90 \%$.

Cheyne-Stokes respiration is usually defined as crescendo-decrescendo periodic respiration with a central apnoea index of $\geqslant 10 /$ hour. Its precise identification is exacting and requires the use of either oesophageal manometry or the simultaneous recording of chest wall impedance and nasal airflow. Apnoeas are invariably associated with oxygen desaturation, and we have previously validated the use of oximetry as a screening tool for Cheyne-Stokes respiration in subjects with heart failure. ${ }^{5}$ In this study a desaturation index of $\geqslant 15$ was found to be highly predictive of Cheyne-Stokes respiration, with a sensitivity of $87 \%$ and a specificity of $81 \%$.

\section{COGNITIVE FUNCTION TESTS}

All tests were performed in a quiet environment between 09.00 and 17.00 hours. Standard protocols with recommended familiarisation test periods were employed. A single investigator (ADS) supervised all the tests on the day before overnight oximetry. No feedback was given to subjects on their level of performance.

National adult reading test

The predicted premorbid intelligence quotient (IQ) was estimated using the national adult reading test (NART). The subject reads aloud a list of 50 words of varying phonetic complexity. The number of phonetic errors is recorded and the predicted IQ is calculated from published tables. Studies in subjects with cortical atrophy have shown that NART is a sensitive marker of premorbid rather than current IQ. ${ }^{7}$

\section{Paced auditory serial addition test}

The rate of information processing and level of attention were assessed using the two second and four second paced auditory serial addition test (PASAT). ${ }^{8}$ An audio tape delivers a list of 61 single digit numbers ranging from 1 to 9 at predetermined rate of presentation (four second and two second time intervals). The subjects were instructed to add together successive pairs of numbers in such a way that each and every number is added only to the one that immediately preceded it in the sequence. In the following example the correct verbal responses are shown in parentheses: $5,3(8), 7$ (10), 4 (11), 2 (6).

Both the two second and the four second tests are sensitive markers of impaired attention and concentration. The four second test is also vulnerable to deficits in short term memory.
Reitan trail making test (part B)

The Reitan test was used as a test of speed of visual search, attention, mental flexibility, and motor function. ${ }^{9}$ In this test the subject was required to connect 25 circles (numbered from 1 to 13 and lettered from A to L) alternating between numbers and letters in incremental and sequential order. Test results were recorded in seconds adjusted for age and level of education..$^{10}$

\section{Vigilance tests}

Vigilance is the ability to maintain concentration while performing a tedious, monotonous, or repetitive task. This was measured on a portable computer using the four choice reaction time test (FCRTT) and the Steer Clear driving simulator. No subject had musculoskeletal hand disability.

FCRTT is a self paced and self administered test measuring reaction time to visual stimuli. ${ }^{11}$ Subjects were instructed to press one of four marked buttons on the computer keyboard, corresponding geometrically to one of four illuminated square shapes on the visual display. The test lasts five minutes and is unsupervised. Responses were analysed to determine the mean reaction time.

Steer Clear is a 30 minute test designed to simulate the mental fatigue experienced during a monotonous motorway drive. ${ }^{12}$ It is not a virtual reality driving simulator; consequently, it does not assume previous driving experience. The program graphically displays a two lane straight highway and a single motor car. Obstacles (steers) intermittently appear on the highway at variable rates of presentation (two second to two minute intervals), which the subject has to avoid hitting by changing lane. The test is unsupervised and lasts 30 minutes, during which time 780 steers are passed.

\section{STATISTICAL ANALYSIS}

All analyses were made using SPSS version 8.0 software. Unless otherwise stated all results are presented as means (SEM). Analysis of variance (ANOVA) was used to assess the level of significance of differences between groups of numerical data. ANOVA testing was only applied to normally distributed data when the equal variance assumption was upheld. Reitan and Steer Clear results underwent $\log _{10}$ transformation before statistical analysis. Post-hoc analysis using the Bonferroni method was employed to allow for multiple statistical testing. Correlations between normally distributed variables were calculated using Pearson's correlation coefficient. All differences were taken to be significant at $\mathrm{p}<0.05$.

\section{Results}

DEMOGRAPHIC CHARACTERISTICS AND

OVERNIGHT OXIMETRY

Table 1 shows that the heart failure patients and the healthy volunteers were matched for age and BMI. The heart failure was ischaemic in origin in $78 \%$ of cases (81/104). All New York Heart Association (NYHA) functional classes were represented (I:II:III:IV, 14:42:40:8). Overall $79 \%$ of subjects had 
Table 1 Demographic characteristics and oximetry results

\begin{tabular}{llll}
\hline & NHV $(n=21)$ & $H F(n=81)$ & HF-CSR $(n=23)$ \\
\hline $\begin{array}{l}\text { Demographic characteristics } \\
\text { Age (years) }\end{array}$ & $66(1.4)$ & $63.8(1)$ & $66.9(1.4)$ \\
$\mathrm{BMI}\left(\mathrm{kg} / \mathrm{m}^{2}\right)$ & $25.7(0.5)$ & $26.8(0.4)$ & $25.9(0.7)$ \\
LV diastolic diameter (cm) & $5.2(0.1)$ & $6.4(0.1) \ddagger$ & $6.6(0.2) \ddagger$ \\
Fractional shortening (\%) & $33.3(1.2)$ & $19.1(1.1) \ddagger$ & $15.7(1.4) \ddagger$ \\
Ejection fraction (\%) & $69.8(3.1)$ & $42.7(2.1) \ddagger^{\star}$ & $28.9(3.2) \ddagger$ \\
Oximetry findings & $4.5(0.6)$ & $6.4(0.4)^{\star}$ & $23.9(1.6) \ddagger$ \\
Desaturation index (/h) & $95.8(0.3)$ & $94.4(0.2) \ddagger$ & $93.7(0.4) \ddagger$ \\
Mean $\mathrm{S}_{\mathrm{A}} \mathrm{O}_{2}(\%)$ & $90(0.8)$ & $85.7(0.7) \dagger$ & $82.9(1.3) \ddagger$ \\
Minimum $\mathrm{S}_{\mathrm{A}} \mathrm{O}_{2}(\%)$ & $0.2(0.1)$ & $3.2(0.9)$ & $8(2.8) \dagger$ \\
\% Recording time with $\mathrm{S}_{\mathrm{A}} \mathrm{O}_{2} \leqslant 90 \%$ & & &
\end{tabular}

All results are mean $(\mathrm{SEM})$.

${ }^{\star} \mathrm{p} \leqslant 0.005 v$ HF-CSR; $\nmid \mathrm{p} \leqslant 0.05 v$ NHV; $\neq \mathrm{p} \leqslant 0.005 v$ with NHV.

$\mathrm{BMI}$, body mass index; HF, heart failure without Cheyne-Stokes respiration; HF-CSR, heart failure with Cheyne- Stokes respiration; LV, left ventricular; NHV, normal healthy volunteers.

Table 2 Cognitive function test results

\begin{tabular}{llll}
\hline & NHV $(n=21)$ & $H F(n=81)$ & HF-CSR (n=23) \\
\hline Premorbid IQ & $113.5(1.9)$ & $107(1.4)$ & $110(2.4)$ \\
Reitan test (s) & $62.4(10.3)$ & $95.1(8)$ & $113.7(24.7)$ \\
PASAT, 2 second (n correct) & $32.5(3.3)$ & $27(1.3)$ & $25.4(2.5)$ \\
PASAT, 4 second (n correct) & $51.1(2)$ & $49(1.1)$ & $45.9(2.1)$ \\
PASAT (total n incorrect) & $35.1(4.5)$ & $45.3(2.2)$ & $48.1(4)$ \\
Reaction time (s) & $0.6(0.05)$ & $0.86(0.03)^{\star}$ & $1(0.07)^{\star}$ \\
Steer Clear (number hit) & $33(4.6)$ & $66.7(6.2)^{\star}$ & $103.2(17.5)^{\star}$ \\
\hline
\end{tabular}

All results are mean (SEM).

${ }^{\star} \mathrm{p} \leqslant 0.005$ v NHV.

$\mathrm{HF}$, heart failure without Cheyne-Stokes respiration; HF-CSR, heart failure with Cheyne-Stokes respiration; NHV, normal healthy volunteers; PASAT, paced auditory serial addition test.

symptoms of mild to moderate severity. The mean (SD) NYHA functional class was 2.4 (0.8). The mean (SD) frusemide (furosemide) and captopril doses were 91.5 (94.8) $\mathrm{mg}$ and 99.8 (65.4) $\mathrm{mg}$, respectively. Eighty two per cent of the patients (85/104) were taking ACE inhibitors. Indices of left ventricular function and overnight oximetry were lower in the patient group.

Ninety per cent of normal healthy volunteers $(19 / 21)$ and $91 \%$ of the heart failure subjects (95/104) (NS) were either current drivers or had previous driving experience.

\section{COGNITIVE FUNCTION}

Table 2 shows the results of cognitive function tests in heart failure patients with and without Cheyne-Stokes respiration and in the normal healthy volunteers. Subjects were matched for premorbid IQ. Subjects with heart failure performed worse than the normal volunteers in vigilance tests. Heart failure was associated with a $48 \%$ slowing in reaction time (0.89 $(0.03) \mathrm{s} v 0.60(0.05) \mathrm{s} ; \mathrm{p} \leqslant 0.005)$ and twice as many collisions on the Steer Clear test (75 (6) v 33 (5); p $\leqslant 0.005)$. The presence of Cheyne-Stokes respiration did not in itself appear to be a marker for impaired vigilance.

The results of univariate analysis of the factors contributing towards impaired vigilance within the heart failure group are shown in table 3. Vigilance measurements were unrelated to both the degree of left ventricular dysfunction assessed by echocardiography and the overnight oximetry findings.

\section{Discussion}

In this study we have shown that patients with medically treated heart failure of mild to moderate severity are cognitively impaired com-
Table 3 Pearson correlation coefficients for vigilance in 104 heart failure patients

\begin{tabular}{lll}
\hline & Reaction time & Log $_{10}$ steert \\
\hline Premorbid IQ & $-0.28^{\star}$ & -0.12 \\
Age & 0.07 & $0.25^{\star}$ \\
Ejection fraction & 0.10 & -0.17 \\
Mean $\mathrm{S}_{\mathrm{A}} \mathrm{O}_{2}$ & -0.06 & -0.12 \\
${\text { Minimum } \mathrm{S}_{\mathrm{A}} \mathrm{O}_{2}}_{\log _{10} \text { desaturation index }}$ & -0.04 & -0.12 \\
\end{tabular}

$\uparrow$ Number of obstacles (steers) hit.

$\star_{\mathrm{p}} \leqslant 0.05$.

pared with healthy normal volunteers. Cognitive dysfunction in heart failure has been little reported. Previous studies in patients with end stage heart failure awaiting cardiac transplantation report an association between the degree of cognitive dysfunction and reduced cardiac output. ${ }^{4}$ In our subjects the severity of cognitive impairment was unrelated to the degree of left ventricular dysfunction assessed by echocardiography. The effect of CheyneStokes respiration on cognitive function in heart failure has not previously been investigated. We were unable to find evidence in support of our original hypothesis that the presence of overnight Cheyne-Stokes respiration was a contributory factor in the pathogenesis of cognitive dysfunction in heart failure.

PATHOPHYSIOLOGY OF COGNITIVE IMPAIRMENT IN HEART FAILURE

We reasoned that Cheyne-Stokes respiration during sleep might lead to cognitive dysfunction as a result of either apnoea related cerebral hypoxia or increased sleep fragmentation. This hypothesis was based upon the observations from earlier studies in healthy volunteers and patients with obstructive sleep apnoea. Hypoxia has been shown to impair cognitive function in normal subjects at altitude ${ }^{1}$ and in chronic obstructive pulmonary disease. ${ }^{13}$ Sleep fragmentation has been shown to increase subjective and objective measurements of daytime sleepiness ${ }^{214}$ and cognitive dysfunction ${ }^{2}$ in normal healthy volunteers.

Obstructive sleep apnoea is associated with cognitive impairment that is reversed by continuous positive airways pressure. ${ }^{15}$ The origin of this impairment is unclear. Findlay reported that nocturnal hypoxia alone predicted impaired cognitive function. ${ }^{16}$ Other investigators report that both hypoxia and sleep fragmentation have a role in generating cognitive dysfunction. Bedard and colleagues found that deficits in global intellectual function could be explained by the degree of hypoxia, while problems with vigilance (using the four choice reaction time test) could not. ${ }^{17}$ Similarly Naegele and associates found that memory deficits were related to apnoea-hypopnoea index (a crude index of sleep fragmentation), whereas frontal lobe deficits could be related to hypoxia.$^{18}$ The origin of the reduced vigilance detected by the Steer Clear test in obstructive sleep apnoea is unclear. Findlay and colleagues showed that test scores were related to both desaturation index $(r=0.55)$ and apnoea-hypopnoea index $(r=0.3) .{ }^{19}$ In contrast, Flemons and colleagues found that test 
scores were unrelated to the severity of oxygen desaturation, apnoea-hypopnoea index, or arousal index. ${ }^{20}$

Why then is cognitive dysfunction related to sleep disordered breathing in obstructive sleep apnoea but not in heart failure with CheyneStokes respiration? The likely explanation is that the underlying mechanism responsible for apnoea generation is different between these two conditions. Consequently the degree of nocturnal hypoxia and sleep fragmentation is more pronounced in untreated obstructive sleep apnoea than it is in Cheyne-Stokes respiration in treated heart failure. Although Cheyne-Stokes respiration was common (prevalence 23/104, 22\%), the levels of oxygen desaturation seen were mild (mean $\mathrm{S}_{\mathrm{A}} \mathrm{O}_{2}$ of $94.2 \%$ and average minimum $\mathrm{S}_{\mathrm{A}} \mathrm{O}_{2}$ of $85 \%$; only five subjects had a mean overnight $\mathrm{S}_{\mathrm{A}} \mathrm{O}_{2}$ of less than 90\%). The cognitive dysfunction observed in our subjects was therefore unrelated to any of the measured indices of nocturnal desaturation.

A single study in end stage heart failure has suggested that cognitive dysfunction was related to cardiac output measured invasively in the catheter laboratory. ${ }^{4}$ We were unable to show a relation between the level of cognitive function and left ventricular function measured by echocardiography. Further investigation into the relation between cognitive function, central haemodynamics, and cerebral blood flow in heart failure would undoubtedly be of interest.

IMPAIRED VIGILANCE AND FITNESS TO DRIVE

The impaired vigilance we observed in our patients with stable, medically treated heart failure of mild to moderate severity was interesting. The consequence of impaired vigilance is well described in obstructive sleep apnoea and narcolepsy. Up to $31 \%$ of patients with untreated obstructive sleep apnoea have a history of sleep related motor vehicle accidents, ${ }^{3}$ and a diagnosis of obstructive sleep apnoea carries an odds ratio of 6.3 for having a road traffic accident. ${ }^{21}$ Findlay and colleagues retrospectively analysed the official Virginia State driving records of subjects with obstructive sleep apnoea according to their Steer Clear performance. ${ }^{19}$ Subjects with untreated obstructive sleep apnoea and "very poor" test performance (defined as hitting $\geqslant 4.5 \%$ of obstacles) had a sevenfold increase in their rate of motor vehicle accidents compared with a group of normal healthy volunteers $(0.38 v$ 0.05 accidents/driver $/ 5$ years; $p \leqslant 0.05)$. The proportion of obstacles hit by our heart failure subjects was $9.6 \%$.

The relation between real life driving and performance test results is unclear, and the ability of such tests to risk stratify patients prospectively for safety to drive is unknown. It is worth repeating that Steer Clear is not a virtual reality driving simulator-it does not have pedals or a steering wheel; it is a crude computer "game", proficiency at which requires the ability to maintain one's concentration under monotonous conditions. What is not a game, however, is that these skills could be important for the performance of tasks such as the operation of machinery and motorway driving. ${ }^{19}$

Safety to drive is a question that has been incompletely addressed in the general heart failure population. The European Society of Cardiology task force report on driving and heart disease merely recommends that heart failure patients should be advised not to drive if they are "symptomatic at rest or at the wheel." "22 The vigilance test results reported in this study are interesting as they raise important questions over safety to drive in a cardiovascular disease that affects $1 \%$ of the British population. Are heart failure patients at increased risk of motor vehicle accidents? If so, what is the precise mechanism at work responsible for their increased risk? The answers to these questions are at present speculative.

\section{CONCLUSIONS}

In this study we found that heart failure patients performed significantly worse than a matched group of healthy normal volunteers in cognitive function tests measuring vigilance. This dysfunction was unrelated to the presence of overnight Cheyne-Stokes respiration. The mechanism responsible for the impaired vigilance is unclear and requires further investigation.

We express our thanks to Mr P Jamie (clinical psychologist) for his expert advice on the selection and use of the neuropsychological tests employed in this study, and to Dr L J Findley for supplying a copy of the Steer Clear driving simulator program.

1 Townes B, Hornbein T, Schoene R, et al. Human cerebral function at extreme altitude. In: West J, Lahiri S, eds. High altitude and man. Bethseda: American Physiological Society, 1984:31-6.

2 Martin SE, Engleman HM, Deary IJ, et al. The effect of sleep fragmentation on daytime function. Am $\mathcal{F}$ Respir Crit Care Med 1996;153:1328-32.

3 Findley LJ, Unverzagt ME, Suratt PM. Automobile Findley LJ, Unverzagt ME, Suratt PM. Automobile
accidents involving patients with obstructive sleep apnoea. Am Rev Respir Dis 1988;138:337-40.

4 Bornstein RA, Starling RC, Myerowitz PD, et al. Neuropornstein RA, Starling RC, Myerowitz PD, et al. Neuro-
psychological function in patients with end-stage heart failure before and after cardiac transplantation. Acta Neurol ure before and after carc $1995 ; 91: 260-5$.

Scand 1995;91:260-5.
5 Staniforth AD, Kinnear WJM, Starling R, et al. Nocturnal desaturation in patients with stable heart failure. Heart 1998;79:394-9.

6 Warley ARH, Stradling JR, Mitchell J. Evaluation of Ohmeda 3700 pulse oximeter. Thorax 1987;42:892-6.

7 Nelson HE, O'Connell A. Dementia: the estimation of premorbid intelligence levels using the new adult reading test. Cortex 1978;14:234-44.

8 Gronwall DMA. Paced auditory serial addition task: a measure of recovery from concussion. Percept Mot Skills 1977;44:367-73.

9 Reitan RM, Wolfson D. The Halstead-Reitan neuropsychological test battery. Tucson: Neuropsychology Press, 1985.

10 Alekoumbides A, Charter RA, Adkins TG, et al. The Alekoumbides A, Charter RA, Adkins TG, et al. The
diagnosis of brain damage by the WAIS, WMS and Reitan diagnosis of brain damage by the WAIS, WMS and Reitan battery using standardized scores corrected for 11 Wilkinson RT, Houghton D. Portable four choice reaction
time test with magnetic tape memory. Behav Res Methods Instrum 1975;7:441-6.

12 Findley L, Fabrizio M, Knight H, et al. Driving simulator performance in patients with sleep apnoea. Am Rev Respir Dis 1989;140:529-30.

13 Grant I, Heaton R, McSweeny A, et al. Brain dysfunction in COPD. Chest 1980;77:308-9.

14 Martin SE, Wraith PK, Deary IJ, et al. The effect of nonvisible sleep fragmentation on daytime function. Am 7 Respir Crit Care Med 1997;155:1596-601.

15 Engleman HM, Martin SE, Deary IJ, et al. Effect of continuous positive airway pressure treatment on daytime function in sleep apnoea/hypopnoea syndrome. Lancet 1994;343:572-5.

16 Findley LJ, Barth JT, Powers DC, et al. Cognitive impairment with obstructive sleep apnoea and associated hypoxaemia. Chest 1986;90:686-90. 
17 Bedard MA, Montplaisir J, Richer F, et al. Obstructive sleep apnoea syndrome: pathogenesis of neuropsycho8 Naegele B, Thouvard V, Pepin JL, et al. Deficits in cognitive executive function in patients with sleep apnoea syndrome. Sleep 1995;18:43-52.

19 Findlay L, Unverzagt M, Guchu R, et al. Vigilance and automobile accidents in patients with sleep apnoea or narcolepsy. Chest 1995;108:619-24.
20 Flemons WW, Remmers JE, Whitelaw WA. The correlation of a computer driving program with polysomnographic indices and neuropsychological tests in consecutively referred patients for assessment of sleep apnea. Sleep 1993; 16: S71.

21 Teran-Santos J, Jimenez-Gomez A, Cordero-Guevara J. The association between sleep apnea and the risk of traffic accidents. N Engl F Med 1999;340:847-51

22 European Society of Cardiology Task Force. Driving and heart disease. Eur Heart $\mathcal{F}$ 1998;19:1165-77.

\section{IMAGES IN CARDIOLOGY}

\section{Complete angiographic view of the coronary-subclavian steal syndrome}

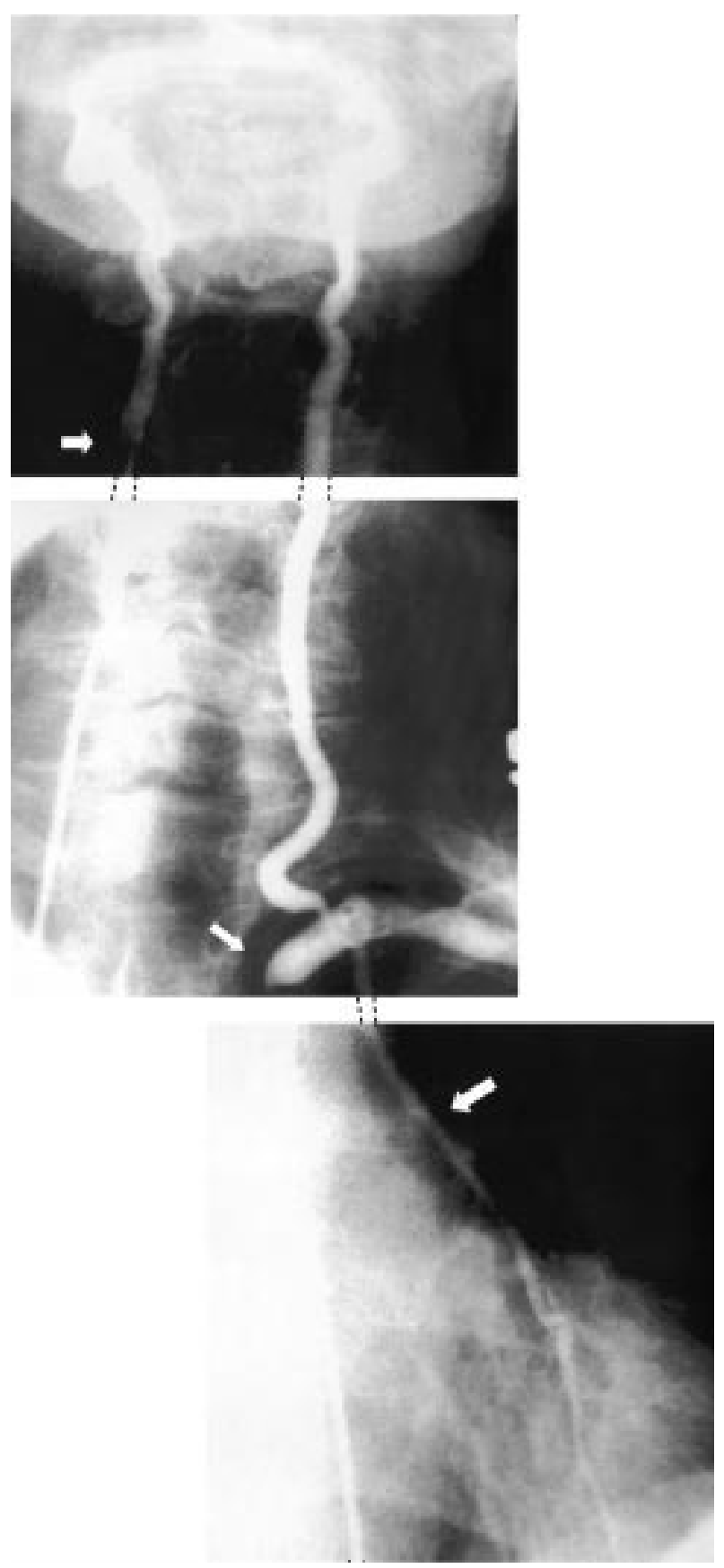

Coronary-subclavian steal syndrome is an uncommon cause of recurrent angina following internal mammary bypass.

A 63 year old patient with history of moderate hypertension, type 2 diabetes, and dyslipidaemia presented with recurrent angina one year after left internal mammary to left anterior descending coronary artery bypass and saphenous vein graft to right coronary artery bypass.

Physical examination showed a murmur in the subclavian region. Transthoracic echocardiography demonstrated normal left ventricle end diastolic volume and ejection fraction and a moderate ipokinesis of the anterior and apical regions of the left ventricle.

The patient underwent coronary and subclavian artery angiography for suspected coronary-subclavian steal syndrome. The coronary angiogram showed a subocclusion of the proximal portion of the left anterior descending coronary artery and reversal flow in the internal mammary artery graft, a severe stenosis of the left circumflex coronary artery, and an occlusion of the middle portion of the right coronary artery. The bypass angiogram revealed the patency of the vein graft. The internal mammary artery was not selectively catheterised because of the proximal occlusion of the left subclavian artery. The internal mammary artery graft was shown by selective angiography of the right vertebral artery and it revealed a collateral flow through the left vertebral artery, to the left subclavian and internal mammary arteries (top, middle and bottom, white arrows). The left vertebral artery had an ostial subcritical stenosis (middle, white arrow). The protection against cerebral ischaemia offered by the vertebral lesion might explain the absence of cerebral symptoms.

The patient underwent percutaneous angioplasty of the subclavian artery, left circumflex artery, and left anterior descending coronary artery, which led to a rapid improvement in the patient's symptoms.

Performance of coronary and brachiocephalic angiography is indicated in recurrent angina in patients with internal mammary artery bypass graft. When possible, revascularisation of the subclavian artery is the treatment of choice for the coronary-subclavian steal syndrome.

GIANLUCA RIGATELLI GIANFRANCO FRANCO GIORGIO RIGATELLI 